\title{
Glucose-6-phosphate dehydrogenase neutralizes stresses by supporting reductive glutamine metabolism and AMPK activation
}

\section{Signal Transduction and Targeted Therapy (2021)6:46}

\section{Dear Editor,}

Glucose-6-phosphate dehydrogenase (G6PD) is the rate-limiting enzyme in the oxidative pentose phosphate pathway (oxPPP) that can generate cytosolic NADPH (Fig. 1a) for biosynthesis and oxidative defence. Here, we reveal a previously unidentified function of G6PD. It, even the natural G6PD deficiency-associated mutant without the activity to maintain the normal oxPPP, can antagonize the stresses by supporting the reductive glutamine metabolism and AMPK activation, independently of the NADPH generation by the oxPPP.

We deleted G6PD in HeLa, MDA-MB-231, and HCT116 cells, and then re-expressed the wild type (WT) enzyme, some natural variants, and mechanism-based inactivated mutants ${ }^{1}$ (Fig. $1 \mathrm{~b}$ and Supplementary Fig. S1a-c). G6PD-KO significantly sensitized cells to hydrogen peroxide $\left(\mathrm{H}_{2} \mathrm{O}_{2}\right)$, hypoxia, and the electron transport chain (ETC) inhibition by antimycin $A$, which, however, was completely rescued by all the expressions except artificial mutants including H263A (Fig. 1c and Supplementary Fig. S1a-c), even though they displayed obviously decreased activities by measuring the product of 6-phosphate gluconolactone (Fig. 1d) and the $\left[3^{-2} \mathrm{H}\right]$-glucose-labeled NADPH $\mathrm{m}+1$ generated from oxPPP (Fig. 1 e and Supplementary Fig. S1d). The NADPH/NADP ${ }^{+}$ratio was dramatically reduced in HeLa ${ }^{\mathrm{KO}}$ cells, but it was also totally reversed by natural mutants but not $\mathrm{H} 263 \mathrm{~A}$ (Fig. 1f). We meantime observed compensatively increased $\left[3{ }^{2} \mathrm{H}\right]$-glucose-labeled $\mathrm{NADPH} \mathrm{m}+2$ in $\mathrm{HeLa}^{\mathrm{KO}}$ and natural mutant-re-expressing cells (Fig. 1e), which resulted from the newly synthesized NADPH incorporating ribose 5-phosphated $\mathrm{m}+2$ derived from the nonoxPPP (Supplementary Fig. S1a). These data suggest that enabling the robust oxPPP is not required for the anti-stress activity of G6PD.

We then performed metabolomic profiling on $\mathrm{HeLa}^{\mathrm{KO}}, \mathrm{HeLa}^{\mathrm{WT}}$, and $\mathrm{HeLa}^{\mathrm{R} 257 \mathrm{G}}$ cells, and found that, except the products of the OxPPP, most of the significantly changed metabolites in HeLa ${ }^{\mathrm{KO}}$ cells were reversed by the re-expression of R257G without detectable activity (Supplementary Fig. S2a-c). Among the significantly increased metabolites in $\mathrm{KO}$ cells, pyruvate and ahydroxyglutarate were associated with glycolysis and center carbon metabolism (Fig. 1g), and the KEGG analysis also enriched the genes involved in these pathways (Supplementary Fig. S2d). Indeed, G6PD-KO promoted glucose uptake and lactate secretion, which were suppressed by re-expressing WT and R257G-G6PD, but not $\mathrm{H} 263 \mathrm{~A}$ mutant (Fig. $1 \mathrm{~h}$ ). The ${ }^{13} \mathrm{C}_{6}$-glucose tracing revealed similar labeled fractions of all the metabolites tested in HeLa ${ }^{\mathrm{WT}}$ and HeLa ${ }^{\mathrm{KO}}$ cells, but increased cellular levels of glycerol 3phosphate, lactate, acetyl-CoA, and a-hydroxyglutarate, and decreased levels of aspartate and all the intermediates in the

\author{
; https://doi.org/10.1038/s41392-020-00399-x
}

tricarboxylic acid cycle except a-ketoglutarate in $\mathrm{HeLa}^{\mathrm{KO}}$ cells (Supplementary Fig. S3a,b). These metabolic changes were exactly similar to those induced by NADH accumulation under ETC dysfunction. ${ }^{2}$ We further measured an increased NADH/NAD ${ }^{+}$ ratio in $\mathrm{HeLa}^{\mathrm{KO}}$ but in $\mathrm{HeLa}{ }^{\mathrm{WT}}$ and $\mathrm{HeLa}^{\mathrm{R} 257 \mathrm{G}}$ cells (Fig. 1i), suggesting that G6PD can maintain the $\mathrm{NADH} / \mathrm{NAD}^{+}$homeostasis, independently of its intact activity.

Hypoxia, $\mathrm{H}_{2} \mathrm{O}_{2}$, and antimycin $A$ treatments increased the $\mathrm{NADH} / \mathrm{NAD}^{+}$ratio (Supplementary Fig. S4a). To determine whether the deregulated $\mathrm{NADH} / \mathrm{NAD}^{+}$homeostasis contributed to cell death in G6PD-KO cells, we used a-ketobutyrate, a pyruvate analogue substantially converting NADH to $\mathrm{NAD}^{+},{ }^{3}$ to reduce the $\mathrm{NADH} / \mathrm{NAD}^{+}$ratio (Fig. $1 \mathrm{j}$ and Supplementary S4b). We found that a-ketobutyrate, as well as other electron accepters, significantly protected G6PD-KO cells against antimycin $\mathrm{A}$ and $\mathrm{H}_{2} \mathrm{O}_{2}$ (Fig. $1 \mathrm{k}$ and Supplementary Fig. S4c).

Furthermore, we expressed a doxycycline-inducible $L b \mathrm{NOX}^{4}$ (Fig. 1l), which transfers electrons from NADH to oxygen, in HeLa ${ }^{\mathrm{KO}}$ cells. Both cytosolic LbNOX and mitochondrial mitoLbNOX significantly decreased $\mathrm{NADH} / \mathrm{NAD}^{+}$ratio (Supplementary Fig. S4d, e) and repressed antimycin A-induced cell death (Fig. 1m). Another enzyme, TPNOX, can convert NADPH to NADP ${ }^{+}$and thus decrease the NADPH/NADP ${ }^{+}$ratio, but it also meantime reduced the NADH/NAD ${ }^{+}$ratio. ${ }^{5}$ Interestingly, only the cytosolic TPNOX, not mitochondrial mitoTPNOX, rescued $\mathrm{HeLa}^{\mathrm{KO}}$ cells (Fig. $1 \mathrm{~m}$ ). Therefore, G6PD-KO sensitizes cells to the deregulated NADH/ $\mathrm{NAD}^{+}$ratio, most likely in the cytosol, not to the decreased $\mathrm{NADPH} / \mathrm{NADP}^{+}$.

Although lipid biosynthesis was thought to require a large amount of NADPH mainly afforded by the oxPPP, we found no difference in lipids and ${ }^{13} \mathrm{C}_{6}$-glucose-labeled fatty acids between $\mathrm{HeLa}{ }^{\mathrm{KO}}$ and HeLaWT cells (Supplementary Fig. S5a, b). However, our tracing results of ${ }^{13} \mathrm{C}_{5}$-glutamine indicated that the metabolic flux of glutamine to fatty acids actually decreased in HeLa ${ }^{\mathrm{KO}}$ cells (Supplementary Fig. S5c), although its contribution was much less than glucose in the normal condition. Glutamine carbon was usually used to synthesize fatty acid through the reductive pathway (Supplementary Fig. S5d), which was specifically promoted by ETC dysfunction. ${ }^{2}$ Our metabolic flux analyses of ${ }^{13} \mathrm{C}_{5}$-glutamine in $\mathrm{HeLa}^{\mathrm{WT}}, \mathrm{HeLa}^{\mathrm{KO}}$, and $\mathrm{HeLa}^{\mathrm{R} 257 \mathrm{G}}$ cells showed that antimycin $\mathrm{A}$ obviously boosted reductive glutamine metabolism in $\mathrm{HeLa}^{\mathrm{WT}}$ and HeLa ${ }^{\mathrm{K} 257 \mathrm{G}}$ but not HeLa ${ }^{\mathrm{KO}}$ cells, indicated by the increased ${ }^{13} \mathrm{C}_{5}$-glutamine-labeled fractions of isocitrate $\mathrm{m}+5$ (Fig. 1n).

Although the fraction and content of ${ }^{13} \mathrm{C}_{5}$-glutamine-derived acetyl-CoA $\mathrm{m}+2$ were not promoted in $\mathrm{HeLa}^{\mathrm{KO}}$ cells by antimycin $A$, the cellular level of acetyl-CoA did not decrease 
b
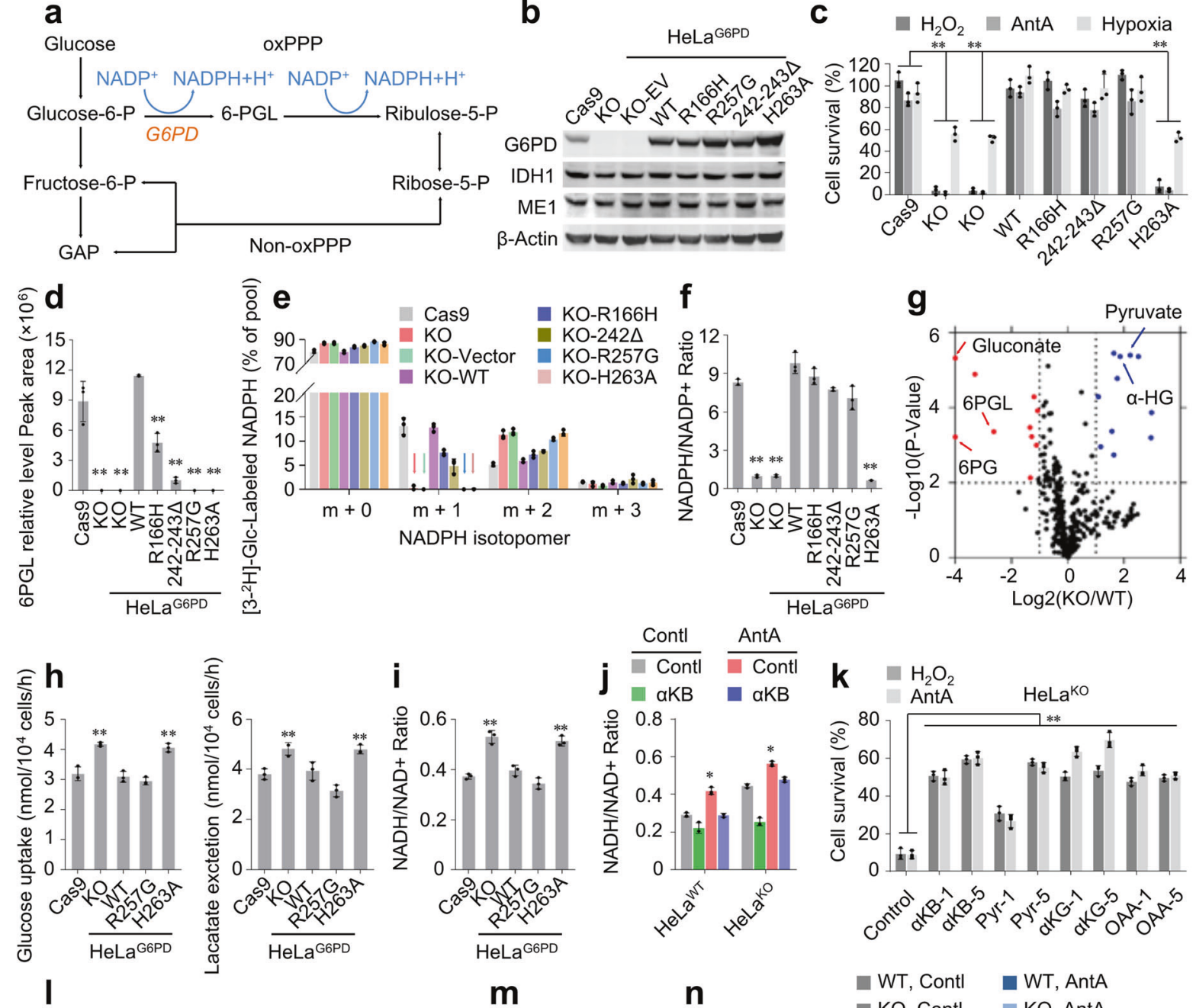

K $\quad \begin{aligned} & \mathrm{H}_{2} \mathrm{O}_{2} \\ & \mathrm{AntA}\end{aligned}$
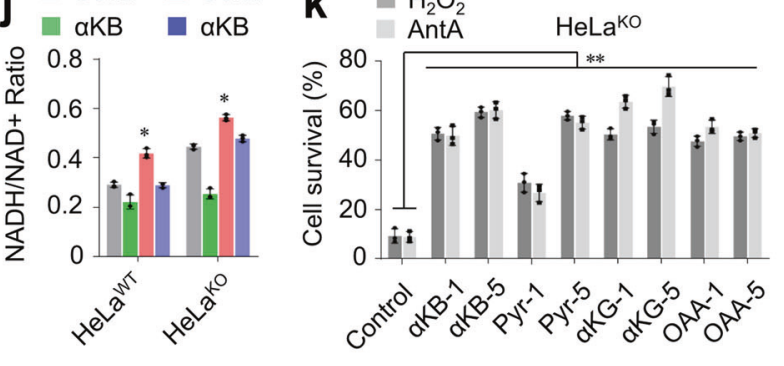

I
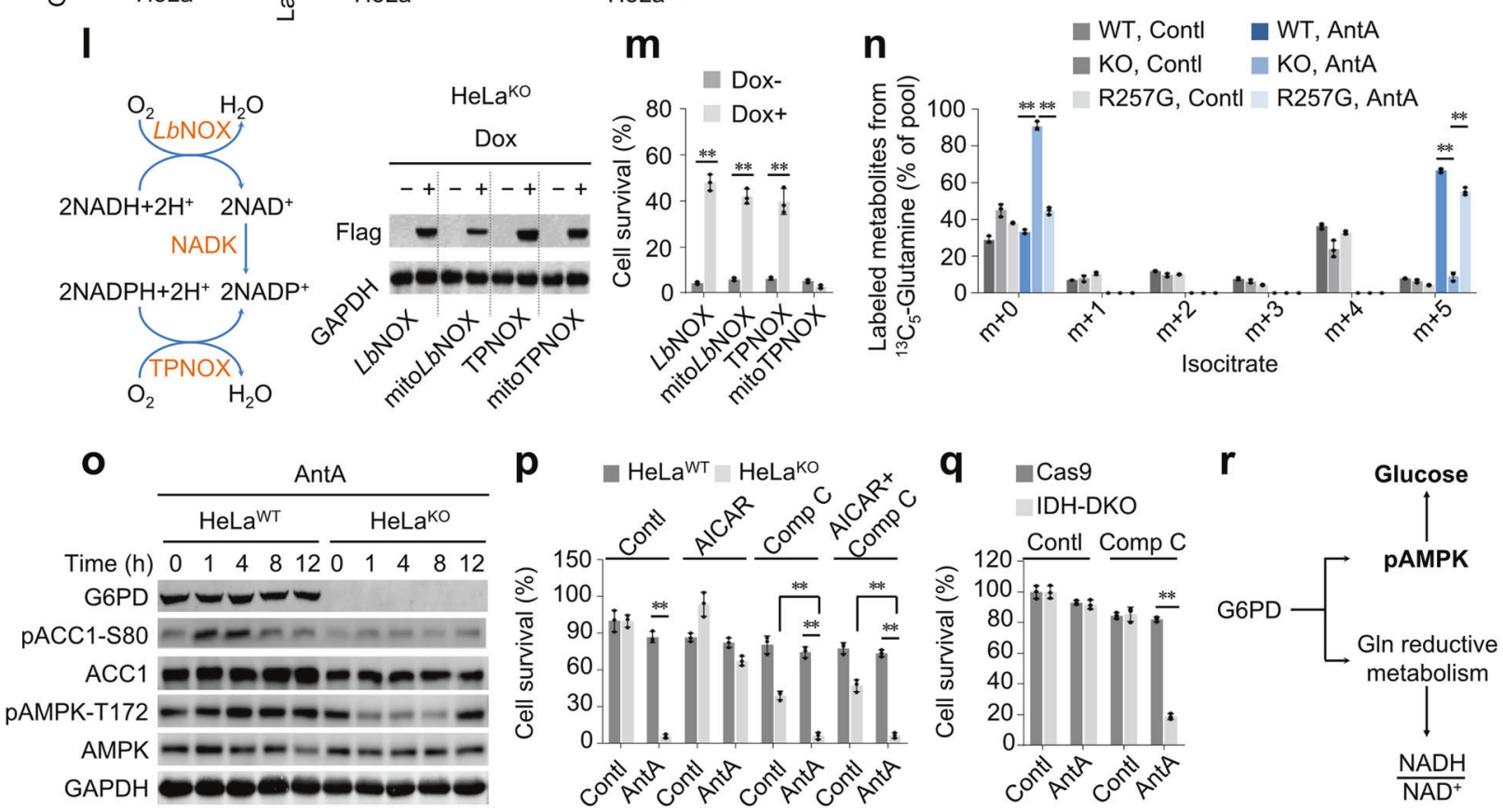

(Supplementary Fig. S5e,f), suggesting the presence of a compensative pathway, most likely from glucose metabolism. As expected, upon the antimycin A treatment, we observed an increase in ${ }^{13} \mathrm{C}_{6}$-glucose-derived malate $m+3$, citrate $m+3$ and $\mathrm{m}+5$, and acetyl-CoA $\mathrm{m}+2$ in HeLa ${ }^{\mathrm{KO}}$ cells (Supplementary Fig. S6a-d), demonstrating that HeLa ${ }^{\mathrm{KO}}$ cells still utilize glucose, instead of glutamine, to synthesize acetyl-CoA even under ETC inhibition. 
Fig. 1 G6PD neutralizes stresses by supporting reductive glutamine metabolism and AMPK activation. a A simple schematic for the oxidative PPP and the non-oxidative PPP. G6PD enzymatic activity-deficiency alleles are well tolerated, except that it could bring a risk of acute nonspherocytic hemolytic anemia triggered by exogenous oxidative stressors in red blood cells. Based on the report from the World Health Organization, G6PD deficiency-associated variants are divided into several types, class-I G6PD mutants with an activity often less than $1 \%$ of normal are associated with chronic NSHA; class-II have an activity less than 10\% of normal; class-III show $10-60 \%$ of residual enzyme activity; class-IV are nearly normally active. Intriguingly, up to date, 85 class-I mutants, accounting for about $45 \%$ of the total, have been identified, but no null mutation has been reported. These observations suggest that G6PD protein could have other function(s) than the mediation of the robust oxPPP, which is indispensable to embryonic development. b Western blots to validate the knockout of G6PD in HeLa cells, and the reexpression of the empty vector (KO-EV), WT G6PD, or G6PD mutants (indicated as HeLa ${ }^{\mathrm{KO}}$, HeLaWT, HeLa ${ }^{\mathrm{R} 166 \mathrm{H}}, \mathrm{HeLa}^{\mathrm{R} 257 \mathrm{G}}, \mathrm{HeLa}{ }^{242-243 \Delta}$, and $\mathrm{HeLa}{ }^{\mathrm{H} 263 \mathrm{~A}}$ ). IDH1 and ME1 were also detected, and $\beta$-actin was used as the loading control. c Survival of HeLa cells with different states of G6PD, as indicated, after treatments either with $100 \mu \mathrm{M} \mathrm{H}_{2} \mathrm{O}_{2}$ for $8 \mathrm{~h}$ or $1 \mu \mathrm{M}$ antimycin $\mathrm{A}$ for $24 \mathrm{~h}$, or under hypoxia $\left(0.5 \% \mathrm{O}_{2}\right)$ for $24 \mathrm{~h}$, normalized to untreated cells. Here, one class-II variant, R166H, and two class-I mutants, $242-243 \Delta$ and R257G, were used. d The relative abundance of cellular 6-phospho-D-glucono-1,5-lactone (6PGL) by LC-MS/MS in HeLa cells with different states of G6PD, as indicated. e Mass isotopomer analysis of NADPH in HeLa cell lines, as indicated, cultured with the medium containing $10 \mathrm{mM}$ of [3- $\left.{ }^{2} \mathrm{H}\right] \mathrm{glucose}$ for $8 \mathrm{~h}$. $\mathrm{f}$ The $\mathrm{NADPH} / \mathrm{NADP}^{+}$ratio in HeLa cells with different states of G6PD, as indicated. g Cellular metabolites were measured by LC-MS/MS with an untargeted metabolomic method. Volcano plot of cellular metabolites in HeLa ${ }^{\mathrm{KO}}$, relative to HeLa ${ }^{\mathrm{WT}}$ cells. $\mathbf{h}$ Glucose uptake (left) and lactate excretion (right) in HeLa cells with different states of G6PD, as indicated, under the normal condition. Data were from triplicate experiments, and all experimental data were verified in at least two independent experiments. $\mathbf{i} \mathrm{NADH} / \mathrm{NAD}{ }^{+}$ratio in HeLa cells with different states of G6PD, as indicated, under the normal condition. $\mathrm{j} N A D H / N A D^{+}$ratio in $\mathrm{HeLa}^{\mathrm{KO}}$ and $\mathrm{HeLa}^{\mathrm{WT}}$ cells treated with antimycin $\mathrm{A}(1 \mu \mathrm{M})$ and/or $\alpha-\mathrm{KB}$ $(2 \mathrm{mM})$ for $4 \mathrm{~h}$. $\mathbf{k}$ Survival of HeLa ${ }^{\mathrm{KO}}$ cells treated with antimycin $\mathrm{A}(1 \mu \mathrm{M})$ or $\mathrm{H}_{2} \mathrm{O}_{2}(100 \mu \mathrm{M})$, without or with $\alpha-\mathrm{KB}(1$ or $5 \mathrm{mM})$, Pyruvate $(1$ or $5 \mathrm{mM}$ ), and OAA ( 1 or $5 \mathrm{mM}$ ) for $24 \mathrm{~h}$, normalized to untreated cells. I Confirmation of Dox-induced expression of $\angle b N O X$, mitoLbNOX, TPNOX, and mitoTPNOX in HeLa ${ }^{\mathrm{KO}}$ cells. $m$ Survival of cell lines from $(\mathrm{G})$ treated with antimycin $\mathrm{A}(1 \mu \mathrm{M})$ in the presence or absence of Dox (50 nM, $24 \mathrm{~h}$ prior to antimycin A treatment) for $24 \mathrm{~h}$, normalized to untreated cells. $\mathbf{n}$ Mass isotopomer analysis of isocitrate in HeLaWT, HeLa ${ }^{\mathrm{KO}}$, and HeLa ${ }^{\mathrm{K} 257 \mathrm{G}}$ cells cultured with ${ }^{13} \mathrm{C}_{5}$-glutamine for $4 \mathrm{~h}$ in the presence of antimycin A $(1 \mu \mathrm{M})$. o Western blot analysis of G6PD, pACC1, ACC1, pAMPK $\alpha$, and AMPK $\alpha$ in HeLa cells with different states of G6PD, as indicated, treated with $1 \mu \mathrm{M}$ antimycin A for the time as indicated. GAPDH was used as the loading control. p Survival of HeLaWT and HeLa ${ }^{K O}$ cells treated without or with $1 \mu M$ antimycin A for $24 \mathrm{~h}$, in the presence or absence of $500 \mu \mathrm{M}$ AICAR (pretreatment for $12 \mathrm{~h}$ ) and/or $10 \mu \mathrm{M}$ compound C. q Survival of HeLa/Cas9 and HeLa ${ }^{\text {IDH-DKÓ }}$ cells treated with $10 \mu \mathrm{M}$ compound $\mathrm{C}$ and/or $1 \mu \mathrm{M}$ antimycin $\mathrm{A}$ for $24 \mathrm{~h}$. $\mathbf{r}$ The brief working model for the anti-stress roles of G6PD. Data were from triplicate experiments, and all experimental data were verified in at least two independent experiments. Error bars represent mean \pm SD. ${ }^{*} p<0.05$; ${ }^{* *} p<0.01$ (Student's $t$-test).

The biosynthetic process of acetyl-CoA from glucose produces $\mathrm{NADH}$, whereas that from glutamine via the reductive pathway did not. ${ }^{2}$ This could underlie the deregulated NADH/NAD ${ }^{+}$ratio in G6PD-KO cells.

We next deleted IDH1, as well as its mitochondrial isoform IDH2, to block reductive glutamine metabolism in HeLa cells, and observed the increased $\mathrm{NADH} / \mathrm{NAD}^{+}$ratio (Supplementary Fig. S7a). However, HeLa ${ }^{I D H 1 / 2-D K O}$ cells did not sensitize to antimycin A (Fig. S7a). It suggests that blocking reductive glutamine metabolism is insufficient to explain the susceptibility of G6PD-KO cells to the stresses.

We then observed that antimycin A quickly induced the phosphorylations of AMPK and its substrate ACC1 in HeLa' and HeLa ${ }^{\mathrm{R} 257 \mathrm{G}}$, but not in HeLa ${ }^{\mathrm{KO}}$ and HeLa ${ }^{\mathrm{H} 263 \mathrm{~A}}$ cells (Fig. 10 and Supplementary Fig. S7b), suggesting that G6PD, independently of its intact activity, can effectively support AMPK activation. Furthermore, 5-amino-4-imidazolecarboxamide ribonucleoside (AICAR) was used to activate AMPK in HeLa, MDA-MB-231, and HCT116 cells, and confirmed that the phosphorylations of AMPK and ACC1 in G6PD-KO cells were much slower than those in G6PDWT cells (Supplementary Fig. S7c). As expected, AICAR pretreatment obviously protected G6PD-KO cells against death induced by antimycin $\mathrm{A}$ or $\mathrm{H}_{2} \mathrm{O}_{2}$ (Fig. $1 \mathrm{p}$ and Supplementary Fig. S7d), which was completely blocked by AMPK inhibitor compound C (Fig. 1p and Supplementary Fig. S7e). These data demonstrate that AMPK activation is also necessary for cells to cope with the stresses.

At last, we found that the increased $\mathrm{NADH} / \mathrm{NAD}^{+}$ratio could delay AMPK activation (Supplementary Fig. S8a-C), and verified that simultaneously blocking AMPK and reductive glutamine metabolism mimicked G6PD knockout in HeLa cells under the stress condition (Fig. 1q). Since the oxPPP products were not significantly detected in cells expressing R257G-G6PD, traces of metabolites derived from the leaking activity, or an alternatively unidentified function, of naturally mutated G6PD could function as signaling molecules to trigger cascades to activate AMPK and reductive glutamine metabolism, which is required to neutralize the stresses (Fig. $1 \mathrm{r}$ and Supplementary Fig. S8d). Taken together, our findings help us to better understand the physiological roles of G6PD and its association with human diseases.

\section{DATA AVAILABILITY}

The data that support the findings of this study are available within the Article and its Supplementary Information or from the corresponding author upon reasonable request.

\section{ACKNOWLEDGEMENTS}

We thank Dr. Xiaohui Liu (Metabolomics Facility at Tsinghua University Branch of China National Center for Protein Sciences, China) for technical help. This study was supported in part by grants from the National Nature Science Foundation of China (81972567 and 81672762 to B.L., 81830087 , U1602221 and 31771516 to C.C., 81802671 and 81872414 to D.J.), Grant CIT\&TCD20190333 from the Support Project of High-level Teachers in Beijing Municipal Universities in the Period of 13th Five-year Plan, Tianjin Natural Science Foundation (No. 18JCQNJC79600), Project of Innovative Research Team of Yunnan Province $(2019 \mathrm{HC} 005)$, and Yunnan Fundamental Research Projects (2019FB112 and 202001AW070018 to D.J.).

\section{AUTHOR CONTRIBUTIONS}

B.L. and C.C. conceived and designed the study; B.Z., D.J. and Y.H. performed the experiments; B.Z. and D.J. analyzed the data; L.L., L.Q., R.Y., and X.J. did some Western blot experiments and created some constructs and cell lines; B.L. drafted the paper; B. L., C.C., B.Z., and D.J discussed and finalized the paper.

\section{ADDITIONAL INFORMATION}

The online version of this article (https://doi.org/10.1038/s41392-020-00399-x) contains supplementary material, which is available to authorized users.

Competing interests: The authors declare no competing interests. 
Benfu Zhong ${ }^{1,2}$, Dewei Jiang ${ }^{3}$, Yang Hong ${ }^{1}$, Lifang $\mathrm{Li}^{1}$, Li Qiu ${ }^{1}$, Ronghui Yang ${ }^{2}$, Xiaohan Jin ${ }^{2}$, Yawen Song ${ }^{1}$, Ceshi Chen ${ }^{3}$ and Binghui $\mathrm{Li}^{1,2}$

${ }^{1}$ Department of Cancer Cell Biology and National Clinical Research Center for Cancer, Tianjin Medical University Cancer Institute and Hospital, Tianjin 300060, P. R. China; ${ }^{2}$ Department of Biochemistry and Molecular Biology, Capital Medical University, Beijing 100069, P. R. China and ${ }^{3}$ Key Laboratory of Animal Models and Human Disease Mechanisms of Chinese Academy of Sciences and Yunnan Province, Kunming Institute of Zoology, Chinese Academy of Sciences, Kunming 650223, China

These authors contributed equally: Benfu Zhong, Dewei Jiang Correspondence: Ceshi Chen (chenc@mail.kiz.ac.cn) or Binghui Li (bli@ccmu.edu.cn)

\section{REFERENCES}

1. Cosgrove, M. S., Naylor, C., Paludan, S., Adams, M. J. \& Levy, H. R. On the mechanism of the reaction catalyzed by glucose 6-phosphate dehydrogenase. Biochemistry 37, 2759-2767 (1998).

2. Liu, M. et al. Inhibiting both proline biosynthesis and lipogenesis synergistically suppresses tumor growth. J. Exp. Med. 217, e20191226 (2020).
3. Sullivan, L. B. et al. Supporting aspartate biosynthesis is an essential function of respiration in proliferating cells. Cell 162, 552-563 (2015).

4. Titov, D. V. et al. Complementation of mitochondrial electron transport chain by manipulation of the NAD+/NADH ratio. Science 352, 231-235 (2016).

5. Cracan, V., Titov, D. V., Shen, H., Grabarek, Z. \& Mootha, V. K. A genetically encoded tool for manipulation of NADP(+)/NADPH in living cells. Nat. Chem. Biol. 13, 1088-1095 (2017).

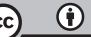

Open Access This article is licensed under a Creative Commons Attribution 4.0 International License, which permits use, sharing, adaptation, distribution and reproduction in any medium or format, as long as you give appropriate credit to the original author(s) and the source, provide a link to the Creative Commons license, and indicate if changes were made. The images or other third party material in this article are included in the article's Creative Commons license, unless indicated otherwise in a credit line to the material. If material is not included in the article's Creative Commons license and your intended use is not permitted by statutory regulation or exceeds the permitted use, you will need to obtain permission directly from the copyright holder. To view a copy of this license, visit http://creativecommons. org/licenses/by/4.0/.

(c) The Author(s) 2021 